\title{
TESE DE SUSZKO E PLURALISMO LÓGICO
}

\section{Edson Bezerra}

\begin{abstract}
RESUMO
A Tese de Suszko (SUSZKO, 1977) afirma a existência de somente dois valores lógicos, o verdadeiro lógico, o verdadeiro e o falso. Neste artigo, argumento em favor da compatibilidade da Tese de Suszko com a tese do Pluralismo Lógico tal como formulado por Hjortland (HJORTLAND, 2011).

Palavras-chave: Lógicas Multivaloradas. Tese de Suszko. Pluralismo Lógico. Consequência Lógica.
\end{abstract}

\begin{abstract}
Suszko's Thesis asserts the existence of only two logical values, the true and the false. In this paper, I argue in favor of the compatibility of Suszko's Thesis and the version of Logical Pluralism formulated by Hjortland (HJORTLAND, 2011).
\end{abstract}

Keywords: Many-valued logics. Suszko’s Thesis. Logical Pluralism. Logical Consequence.

\section{Introdução}

Neste artigo, argumentarei em favor da compatibilidade da Tese de Suszko com a tese do pluralismo lógico, que defende a existência de mais de uma lógica correta. As lógicas multivaloradas são lógicas que diferem da lógica clássica por admitirem valores além dos valores verdadeiro e falso. De acordo com Kneale \& Kneale (KNEALE \& KNEALE, 1962), tais lógicas surgiram a partir dos trabalhos independentes de Łukasiewicz, Post e Bernays. Bernays, em 1919, utiliza tabelas de verdade multivaloradas para provar a independência dos axiomas da lógica proposicional clássica. Embora seja discutível se Bernays pode ser considerado como um dos pioneiros das lógicas multivaloradas, uma vez que ele usou tabelas multivaloradas somente como

\footnotetext{
${ }^{1}$ Mestre e doutorando da Universidade Estadual de Campinas (Unicamp). Este trabalho foi financiado pela Coordenação de Aperfeiçoamento de Nível Superior (CAPES), código 001. E-mail: edson.vinber92@gmail.com. ORCID: 0000-0002-0865-5197.
} 
técnicas para provar um resultado metateórico, sua contribuição para as investigações posteriores em lógicas multivaloradas é indiscutível. ${ }^{2}$ As investigações de Łukasiewicz, em 1920, acerca do problema dos futuros contingentes deram origem a uma série de lógicas multivaloradas. Segundo ele, enunciados sobre o futuro não podem ser verdadeiros nem falsos, mas possíveis. Uma das lógicas resultantes dessa análise é a lógica trivalorada $Ł_{3}$, que inclui o possível como um valor de verdade. Em 1921, Post introduz o método de tabelas de verdade como um procedimento de decisão para lógicas proposicionais. Dada a generalidade de sua investigação, ela abrange casos arbitrários de $n$ valores. De maneira geral, podemos dizer que as lógicas multivaloradas podem ser vistas não somente como lógicas, mas também como um método de investigação metateórica. Uma vantagem dessas lógicas é a de preservarem o aspecto composicional em sua semântica, o que facilita a demonstração de resultados de correção e completude.

A Lógica do Paradoxo (LP) (PRIEST, 1979) é uma lógica multivalorada frequentemente utilizada nas investigações dos paradoxos semânticos, dentre os quais o paradoxo do mentiroso é um dos mais conhecidos. Tarski (TARSKI, 1977) mostra que a introdução de um predicado $\operatorname{Tr}()$ de sentença verdadeira na linguagem objeto de uma teoria $\mathbf{T}$ capaz de expressar aritmética permite a formulação da sentença do mentiroso $\lambda$, que assere sua própria falsidade. Ademais, a presença de tal sentença acarreta uma contradição da forma $\operatorname{Tr}([\lambda])$ e não- $\operatorname{Tr}([\lambda])$. Se $\mathbf{T}$ for uma teoria que possui a lógica clássica como sua base, então $\mathbf{T}$ é trivial em virtude do Princípio da Explosão $\left(P E, \varphi, \neg \varphi \vDash_{\mathbf{T}}\right.$ $\psi)$. Por outro lado, se T tiver LP como lógica de base, então a contradição acarretada por $\lambda$ não implica trivialidade. ${ }^{3}$ Por conseguir lidar não trivialmente com contradições, LP é uma boa candidata para ser base de teorias que possuem contradições, mas que não são triviais.

Embora LP fora escolhida como exemplo, existe uma variedade de casos onde lógicas multivaloradas são utilizadas como ferramentas de análise de diversos problemas, tanto de natureza puramente formal quanto os de natu-

${ }^{2}$ Como Carnielli (CARNIELLI, 2012) ressalta, os trabalhos de Bernays contribuíram fortemente para o desenvolvimento das semânticas algébricas.

${ }^{3}$ Em (PRIEST, 2006a), o leitor encontrará propostas de aplicações de LP em teorias contraditórias, tais como a própria teoria cantoriana de conjuntos. 
reza filosófica. O sucesso dessas lógicas pode sugerir a existência de mais valores de verdade além de verdade e falsidade. Nesse contexto, a Tese de Suszko surge como uma resposta a essa questão. Partindo da constatação de que o conjunto de valores de verdade dessas lógicas é bipartido, para que seja possível definir os conceitos de tautologia e consequência lógica, Suszko (SUSZKO, 1977) afirma que existem somente dois valores lógicos: verdadeiro e falso. Os muitos valores de verdade dessas lógicas são entendidos por ele como valores algébricos, entendidos como sendo os referentes admissíveis das fórmulas. Como contraparte técnica à tese filosófica, Suszko mostra ser possível apresentar uma semântica de bivalorações para essas lógicas, mostrando o caráter bivalente dessas lógicas de um ponto de vista técnico.

A possibilidade de reduzir os muitos valores algébricos aos valores lógicos verdade e falsidade pode sugerir que as lógicas multivaloradas não sejam interessantes de um ponto de vista filosófico e que, além disso, o caráter bivalente dessas lógicas implica a existência de somente uma lógica, a lógica clássica. Neste artigo, argumentarei que isso não é o caso. Ou seja, argumentarei que o caráter bipartite dessas lógicas não implica no monismo lógico. Em particular, argumentarei em defesa da compatibilidade da Tese de Suszko com a proposta do pluralismo lógico tal como formulado por Hjortland (HJORTLAND, 2011).

\section{Lógicas Multivaloradas e Tese de Suszko.}

Nesta seção, apresentarei a teoria das matrizes lógicas, tendo a lógica trivalorada LP (PRIEST, 1979) como objeto de análise. Em seguida, apresentarei a Tese de Suszko e um resultado de redução a bivalorações delineado por Suszko em (SUSZKO, 1977) para o caso de LP. Apresentarei uma possível objeção à Tese de Suszko bem como uma possível resposta. Por fim, argumentarei em defesa da plausibilidade da Tese de Suszko.

\subsection{Lógicas Multivaloradas: linguagem e semântica}

Nesta seção introduziremos informalmente alguns conceitos básicos de lógica proposicionais. Seja $\mathbf{L}$ uma lógica. A linguagem $L$ de $\mathbf{L}$ é um con- 
junto $L=\{A t, \neg, \rightarrow\}$ onde $A t=\left\{p_{i} \mid i \in \mathbb{N}\right\}$ é um conjunto de variáveis proposicionais, ou proposições atômicas, $\neg$ é o conectivo unário de negação e $\rightarrow$ é o conectivo binário de implicação. ${ }^{4} \mathrm{O}$ conjunto de fórmulas For $^{L}$ é definido como segue. Para $\varphi, \psi \in$ For $^{L}$. $^{\prime}$

$$
p_{i}|\neg \varphi| \varphi \rightarrow \psi
$$

A semântica para as lógicas multivaloradas é definida a partir de uma generalização da semântica de tabelas de verdade para a lógica proposicional clássica. Essa generalização deu origem à teoria das matrizes lógicas, que foi desenvolvida a partir dos trabalhos de Łukasiewicz, Tarski e Lindenbaum (TARSKI, 1977). ${ }^{6}$ Uma interpretação para a linguagem $L$ é uma matriz $\mathscr{M}=$ $(V, \neg, \rightarrow, D)$, onde $V$ é um conjunto de valores algébricos, $\neg, \rightarrow$ são operações sobre $V$, e $D \subset V$ o conjunto de valores distinguidos. ${ }^{7}$ Uma valoração é uma função $v: A t \rightarrow V$ do conjunto de variáveis proposicionais no conjunto $\mathrm{V}$ de valores algébricos. A valoração $v$ é estendida recursivamente para $v: F_{0}{ }^{L} \rightarrow V$ como segue. Sejam $f$ e $h$ duas funções, então: $v(\neg \varphi)=f(v(\varphi)) ; v(\varphi \rightarrow \psi)=$ $h(v(\varphi), v(\psi))$. As definições de $f$ e $h$ são especificadas de acordo com a lógica. O conjunto de todas as valorações $v:$ For $^{L} \rightarrow V$ constitui a semântica de $L$, denotado por $\operatorname{sem}_{\mathbf{L}}$. Dizemos que uma valoração $v \in \operatorname{sem}_{\mathbf{L}}$ é modelo para fórmula $\varphi \in$ For $^{L}$ se $v(\varphi) \in D$. Seja $\Gamma \subseteq$ For $^{L}$ um conjunto de fórmulas. Dizemos que $v$ é modelo de $\Gamma$ se $v(\varphi) \in D$, para todo $\varphi \in \Gamma$. Dizemos que $\varphi$ é consistente se $v(\varphi) \in D$, para alguma $v \in \operatorname{sem}_{\mathbf{L}}$. Dizemos que $\varphi$ é uma tautologia se $v(\varphi) \in D$, para toda $v \in \operatorname{sem}_{\mathbf{L}}$. Dizemos que $\varphi$ é uma contradição se $v(\varphi) \notin D$, para toda $v \in s e m_{\mathbf{L}}$. A relação $\vDash_{\mathbf{L}} \subseteq \wp\left(F o r^{L}\right) \times F o r^{L}$ de consequência lógica é

\footnotetext{
${ }^{4}$ Neste trabalho optamos por utilizar linguagens que possuem somente implicação e negação como primitivos, uma vez que esses conectivos tomados como primitivos abrangem uma classe considerável de lógicas multivaloradas. Claramente, uma generalização para quaisquer conectivos é imediata. Para uma apresentação mais geral, convidamos o leitor a consultar (WÓJCICKI, 2013).

${ }^{5}$ Daqui em diante utilizaremos as variáveis proposicionais sem seus índices por simplicidade de escrita.

${ }^{6} \mathrm{O}$ artigo onde a teoria das matrizes lógicas é apresentada se chama Investigations into the Sentential Calculus, p. 38 - 60.

${ }^{7}$ Propositalmente, utilizamos os mesmos símbolos para os conectivos e as operações para ressaltar que as operações são as contrapartes semânticas dos conectivos.
} 
definida como segue: dizemos que $\Gamma \vDash_{\mathbf{L}} \varphi$ se e somente se todo modelo de $\Gamma$ é também modelo de $\varphi$. De acordo com Marcos (MARCOS, 2009), as matrizes lógicas preservam a verofuncionalidade das lógicas multivaloradas. Uma lógica é verofuncional se sua semântica é tal que o valor de verdade de uma fórmula complexa é determinado pelos valores de verdade das fórmulas simples que a compõem.

Dizemos que uma lógica $\mathbf{L}$ é multivalorada se a cardinalidade do conjunto $V$ é maior que dois. Por outro lado, devemos prestar atenção ao seguinte detalhe. Pode ser o caso que uma mesma lógica $\mathbf{L}$ seja caracterizada por matrizes $\mathscr{M}_{1}=\left(V_{l}, \neg, \rightarrow, D_{1}\right)$ e $\mathscr{M}_{2}=\left(V_{2}, \neg, \rightarrow, D_{2}\right)$ tais que $V_{l}$ possui $n$ elementos e $V_{2}$ possui $m$ elementos tais que $n$ e $m$ são diferentes. Assim, a mesma lógica possuiria $n$ e $m$ valores, o que pode soar absurdo. Isso se deve ao fato de que a uma lógica não está associada somente uma matriz, mas uma classe de matrizes. Para resolver esse aparente dilema, Marcos (MARCOS, 2009) chama uma lógica $\mathbf{L}$ de genuinamente $n$-valorada se $n$ é a menor cardinalidade do conjunto $V$ da matriz $\mathscr{M}=(V, \neg, \rightarrow, D)$ associada à lógica $\mathbf{L}$. Dada essas definições, podemos considerar uma lógica $\mathbf{L}$ como um par $\mathbf{L}=(L$, $\vDash_{\mathbf{L}}$ ) onde $L$ é a linguagem de $\mathbf{L}$ e $\vDash_{\mathbf{L}}$ é a relação de consequência semântica definida no conjunto de fórmulas $\mathrm{For}^{L}{ }^{8}{ }^{8}$ Como podemos ver, a distinção entre os valores distinguidos e não-distinguidos é necessária para que os conceitos de tautologia e consequência lógica sejam definidos.

De acordo com Łoś \& Suszko (ŁOŚ \& SUSZKO, 1958), a definição da relação de consequência lógica como preservação de valor de distinguidos implica que a relação $\vDash_{\mathbf{L}}$ tenha as seguintes propriedades:

(I) $\Gamma \cup\{\varphi\} \vDash_{\mathbf{L}} \varphi$ (Reflexividade)

(II) Se $\Gamma \vDash_{\mathbf{L}} \varphi$ e $\Gamma \subseteq \Delta$, então $\Delta \vDash_{\mathbf{L}} \varphi$ (Monotonicidade)

(III) $\operatorname{Se} \Gamma \vDash_{\mathbf{L}} \varphi$ e $\Delta, \varphi \vDash_{\mathbf{L}} \psi$, então $\Delta, \Gamma \vDash_{\mathbf{L}} \psi$ (Corte)

\footnotetext{
${ }^{8}$ Claramente, uma definição de lógica mais geral poderia ser dada, não se restringindo à consequência semântica. De fato, é mais usual trabalhar com uma relação de consequência geral $\Vdash$. Aqui, por questões de simplicidade, optamos por trabalhar somente com a relação de consequência semântica $\vDash$. De todo modo, ambos $\Vdash$ e $\vDash$ são sempre definidos a partir do conjunto de fórmulas de uma lógica $\mathbf{L}$. Uma exposição mais geral da relação de consequência pode ser encontrada em (MALINOWSKI, 1993).
} 
A demonstração desses enunciados é simples. A reflexividade garante que qualquer fórmula é consequência lógica de si própria. A monotonicidade preserva a consequência lógica mediante superconjuntos. E o corte garante, de certo modo, a transitividade da relação de consequência. Dizemos que uma relação de consequência é tarskiana se satisfaz as propriedades (I)-(III). Considere agora um endomorfismo $\varepsilon: A t \rightarrow F^{L} r^{L}$ que atribui para cada variável proposicional uma fórmula. Um endomorfismo é basicamente um mapeamento de uma estrutura em si própria. Claramente, $\varepsilon$ pode ser estendido para todas as fórmulas, de modo que $\varepsilon: F o r^{L} \rightarrow F^{L} r^{L}$. Seja $\Gamma$ um conjunto de fórmulas. Por $\varepsilon(\Gamma)$ entendemos o conjunto $\varepsilon(\Gamma)=\{\varepsilon(\gamma) \mid \gamma \in \Gamma\}$, obtido a partir da aplicação do endomorfismo $\varepsilon$ nas fórmulas do conjunto $\Gamma$. Se além das propriedades $(I)-(I I I), \vDash_{\mathbf{L}}$ satisfizer a propriedade

(IV) $\Gamma \vDash_{\mathbf{L}} \varphi$ se e somente se (sse) $\varepsilon(\Gamma) \vDash_{\mathbf{L}} \varepsilon(\varphi)$ (Estruturalidade)

ela é chamada de tarskiana estrutural. Mostraremos agora que $\vDash_{L}$ é estrutural:

Teorema 2.1. $\vDash_{L}$ é estrutural.

Prova: Assuma $\Gamma \vDash_{\mathrm{L}} \varphi$ e suponha $\varepsilon(\Gamma) \not_{L} \varepsilon(\varphi)$. Então existe uma valoração $v$ $\in \operatorname{sem}_{\mathbf{L}}$ tal que $v(\varepsilon(\gamma)) \in D$, para todo $\varepsilon(\gamma) \in \varepsilon(\Gamma)$, e $v(\varepsilon(\varphi)) \notin D$. Uma vez que $\varepsilon$ é um endomorfismo em $L, v(\gamma) \in D$, para todo $\gamma \in \Gamma$, e $v(\varphi) \notin D$. O que contradiz nossa assunção inicial. $\operatorname{Logo}, \varepsilon(\Gamma) \vDash_{\mathrm{L}} \varepsilon(\varphi)$. O raciocínio converso é similar. Q.E.D.

A estruturalidade preserva a relação de consequência mediante substituição uniforme das variáveis proposicionais que ocorrem nas fórmulas de $\Gamma \mathrm{e}$ em $\varphi$. Ou seja, a definição bipartite do conjunto $V$ de valores de verdade da matriz $\mathscr{M}$ determina que $\vDash_{\mathbf{L}}$ seja tarskiana estrutural. A definição da matriz é bipartite no sentido de todo valor de verdade ser ou não distinguido: para todo $\mathrm{v} \in V, \mathrm{v} \in D$ ou $\mathrm{v} \notin D$. Considere o seguinte exemplo: 
Exemplo 2.2. A Lógica do Paradoxo (LP) (PRIEST, 1979) é apresentada a partir da matriz $\mathscr{M}=(\{1,1 / 2,0\}, \neg, \rightarrow,\{1,1 / 2\})$ cujas operações possuem as seguintes tabelas de verdade:

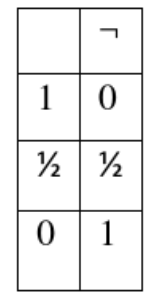

\begin{tabular}{|l|l|l|l|}
\hline$\rightarrow$ & 1 & $1 / 2$ & 0 \\
\hline 1 & 1 & $1 / 2$ & 0 \\
\hline $1 / 2$ & 1 & $1 / 2$ & $1 / 2$ \\
\hline 0 & 1 & 1 & 1 \\
\hline
\end{tabular}

As valorações de LP são definidas como segue:

$$
\begin{aligned}
& v(\neg \varphi)=1-v(\varphi) \\
& v(\varphi \rightarrow \psi)=\text { máximo }(1-v(\varphi), v(\psi))
\end{aligned}
$$

O conjunto de todas as valorações $v:$ For $^{L} \rightarrow\{1,1 / 2,0\}$ constitui a semântica de LP. A definição da relação $\vDash_{L P}$ é similar à definição que demos acima. Não apresentaremos aqui um sistema dedutivo para LP por não ser necessário para os nossos propósitos. O leitor encontrará um sistema de tablôs completo e correto em relação à matriz $\mathscr{M}$ de LP em (PRIEST, 2008). Uma vez que a definição de $\vDash_{\mathrm{LP}}$ como preservando valores distinguidos, as propriedades de $\vDash_{L P}$ são exatamente as descritas acima. Ou seja, $\vDash_{L P}$ é reflexiva, monotônica, possui corte e é estrutural. A enumeração dessas propriedades será importante ao abordamos adiante o resultado de redução de Suszko.

\subsection{Dois valores: Tese e Teorema de Suszko}

Como dissemos anteriormente, as lógicas multivaloradas foram propostas de análise de muitos problemas que a lógica clássica, bivalorada, supostamente não é adequada para lidar por ser muito forte. Por exemplo, diversas lógicas multivaloradas foram propostas para solucionar o problema da antinomia do mentiroso uma vez que a presença dessa sentença em uma teoria clássica acarreta contradições. Dentre elas, as mais conhecidas são a lógicas 
trivaloradas $_{3}$ de Łukasiewicz, $\mathrm{B}_{3}$ de Bochvar, $\mathrm{K}_{3}$ de Kleene e LP de Asenjo/Priest. ${ }^{9} \mathrm{O}$ problema dos futuros contingentes foi também objeto de análise das lógicas multivaloradas. Segundo Łukasiewicz, sentenças sobre o futuro tais como amanhã haverá uma batalha naval não são verdadeiras nem falsas. Portanto, o terceiro excluído não vale irrestritamente. Como uma proposta de análise, Łukasiewicz introduz o sistema $Ł_{3}$ onde o terceiro valor, representado numericamente por $1 / 2$, é interpretado como possível. Łukasiewicz defende que sentenças sobre o futuro devem receber o valor $1 / 2$ ao invés de 1 ou 0 por serem possíveis e não necessárias. Por último, damos como exemplo uma aplicação interessante das lógicas multivaloradas. Por volta dos anos de 1919, Bernays utiliza tabelas de verdade com quatro valores para provar a independência dos axiomas da lógica proposicional clássica. De certo modo, Bernays pode ser creditado como um dos criadores da teoria das matrizes lógicas. Entretanto, essa teoria é creditada a Łukasiewicz, Tarski e Lindenbaum pelo trabalho mais sistemático acerca das matrizes.

Como vimos, as lógicas multivaloradas possuem aplicações filosóficas e técnicas interessantes. Por outro lado, é legítimo questionar se a estipulação de mais valores de verdade além do verdadeiro e do falso é suficiente para invalidar o Princípio de Bivalência, segundo o qual toda sentença é verdadeira ou falsa. Suszko (SUSZKO, 1977) observa que a distinção entre valores distinguidos e não-distinguidos mostra que as lógicas multivaloradas são, na verdade, bivalentes. Como podemos ver na seção anterior, os conceitos de tautologia e consequência lógica são definidos, respectivamente, como uniformidade de valores distinguidos e preservação de valores distinguidos das premissas para a conclusão. Suszko argumenta que existe uma diferença entre valores algébricos e valores lógicos. Os valores algébricos são entendidos como referentes admissíveis de fórmulas, enquanto os valores lógicos são o verdadeiro e o falso. Tomando a lógica LP como exemplo, os valores $1,1 / 2$ e 0 são valores algébricos e o verdadeiro e o falso são os valores lógicos. Ele defende que os valores algébricos e lógicos são de natureza completamente dis-

\footnotetext{
${ }^{9}$ Uma boa exposição de lógicas multivaloradas pode ser encontrada em Malinowski (MALINOWSKI, 1993). Embora a lógica LP seja mais conhecida a partir dos trabalhos de Priest, ela foi elaborada por Asenjo em (ASENJO, 1966).
} 
tinta. Em virtude desse caráter dicotômico dos valores, Suszko afirma que toda lógica é logicamente bivalorada. Essa última afirmação tornou-se conhecida como Tese de Suszko.

Suszko defende que a multiplicação de valores de verdade promovida por Łukasiewicz ocasionou na abolição do Axioma Fregeano (AF), que Suszko formula como segue:

(AF) Todas as sentenças verdadeiras (respectivamente, falsas) possuem um referente em comum.

De acordo com (AF), todas as sentenças verdadeiras referem ao verdadeiro e as falsas referem ao falso. Considerando novamente LP podemos nos perguntar em que sentido ela viola esse axioma. As matrizes de LP tomam 1 e $1 / 2$ como valores distinguidos e 0 como não-distinguido. Assim, nem todas as sentenças verdadeiras terão o verdadeiro como referente, pois uma sentença $\varphi$ pode ter 1 ou $1 / 2$ como referente. No caso da lógica proposicional clássica, (AF) permanece intacto, pois os referentes admissíveis das fórmulas colapsam com seus valores lógicos. E esse é exatamente o ponto no qual as lógicas multivaloradas divergem da clássica. Nesse aspecto, as fórmulas exercem um duplo valor semântico, pois nem sempre seus referentes admissíveis correspondem aos valores lógicos.

É importante esclarecer que Suszko não endossa (AF). Segundo Malinowski (MALINOWSKI, 1985), Suszko rejeita (AF) ao defender que os referentes das sentenças são estados de coisas em vez de valores de verdade. Os estados de coisas são entendidos de acordo com a perspectiva wittgensteiniana em (WITTGENSTEIN, 2010), que podemos interpretar como uma combinação de objetos. Por outro lado, Suszko defende que sentenças podem somente ser verdadeiras ou falsas. Em decorrência da diferença entre o que sentenças referem e quais são seus valores lógicos, Suszko defende que (AF) promoveu uma confusão a esse respeito. E tal confusão levou Łukasiewicz a introduzir a possibilidade como um valor lógico. 
É importante neste ponto dizer que a Tese de Suszko por si não é suficiente para mostrar que toda lógica é logicamente bivalorada. Deve ser mostrado, ainda, que uma tal redução é possível. Embora Suszko não apresente um método construtivo para provar que toda lógica possui dois valores lógicos, no artigo (SUSZKO, 1977) ele sugere que todos os valores distinguidos sejam associados ao valor verdadeiro $(\mathrm{T})$ e que todos os valores não-distinguidos sejam associados ao falso $(\mathrm{F})$. Em outras palavras, dada uma matriz $\mathscr{M}_{1}=$ $\left(\left\{\mathrm{v}_{1}, \ldots, \mathrm{v}_{\mathrm{n}}\right\}, \neg, \rightarrow,\left\{\mathrm{v}_{1}, \ldots, \mathrm{v}_{\mathrm{k}}\right\}\right)$ para $\mathrm{k}<\mathrm{n}$, associamos todos os valores $\mathrm{v}_{1}$, $\ldots, \mathrm{V}_{\mathrm{k}}$ ao valor $\mathrm{T}$ e os demais valores $\mathrm{v}_{\mathrm{k}+1}, \ldots, \mathrm{v}_{\mathrm{n}}$ ao $\mathrm{F}$. Mais adiante discutiremos os efeitos dessa redução à bivalência. ${ }^{10}$

Definição 2.3. Uma bivaloração $b$ é uma $b: F^{L} r^{L} \rightarrow\{\mathrm{T}, \mathrm{F}\}$ definida pelas seguintes cláusulas:

(i) $b(\varphi \rightarrow \psi)=\mathrm{T}$ se e somente se $b(\neg \varphi)=\mathrm{T}$ ou $b(\psi)=\mathrm{T}$

(ii) $b(\varphi \rightarrow \psi)=\mathrm{F}$ sse $b(\varphi)=\mathrm{T}$ e $b(\psi)=\mathrm{F}$

(iii) $b(\neg(\varphi \rightarrow \psi))=\mathrm{T}$ sse $b(\varphi)=\mathrm{T}$ e $b(\neg \psi)=\mathrm{T}$

(iv) $b(\neg \neg \varphi)=\mathrm{T} \operatorname{sse} b(\varphi)=\mathrm{T}$

(v) se $b(\neg \varphi)=\mathrm{T}$ então $b(\varphi)=\mathrm{F}$ ou $b(\varphi)=\mathrm{T}$

(vi) se $b(\neg \varphi)=\mathrm{F}$ então $b(\varphi)=\mathrm{T}$

Dizemos que $\Gamma \vDash^{2}{ }_{\text {LP }} \varphi$ se e somente se toda bivaloração $b$ tal que $b(\gamma)$ $=\mathrm{T}$, para todo $\gamma \in \Gamma$ implica $b(\varphi)=\mathrm{T}$.

Antes de prosseguirmos, é importante esclarecer que o fato de haver uma semântica bivalorada para uma lógica multivalorada não constitui um paradoxo. Como observam Caleiro et al (CALEIRO et al, 2007), embora pareça estranho ouvir que uma lógica multivalorada $\mathbf{L}$ é bivalorada, é necessário perceber que existe uma troca. A semântica multivalorada $\mathbf{L}$ é verofuncional. $\mathrm{Ou}$ seja, o valor de verdade de $\varphi$ é determinado pelos valores de verdade de suas ${ }^{10}$ Como dissemos em uma nota de rodapé anterior, estamos trabalhando em uma perspecti-
va totalmente semântica. Os resultados nos quais Suszko se baseia são provenientes da lógica
abstrata, que basicamente considera uma lógica $\mathbf{L}$ como um par $(L, \Vdash)$, onde $L$ é uma lingua-
gem e $\Vdash$ é uma relação de consequência definida a partir das fórmulas de $L$. Aqui, por como-
didade, adotaremos uma perspectiva totalmente semântica, estando cientes de que nossa
construção é possível em decorrência de resultados mais gerais acerca de lógicas abstratas. 
subfórmulas. No caso da semântica bivalorada para LP é fácil ver que a verdade de $\neg \varphi$ não é determinado pela falsidade de $\varphi$. Ou seja, $b$ não é verofuncional. Portanto, a transição de uma semântica verofuncional para uma semântica bivalorada para uma lógica multivalorada $\mathbf{L}$ tem como preço a perda da verofuncionalidade. Mostraremos agora que a semântica definida na Definição 2.3 é equivalente à semântica multivalorada de LP.

Teorema 2.4. Dada uma bivaloração $b: F^{L} r^{L} \rightarrow\{\mathrm{T}, \mathrm{F}\}$, existe uma valoração $v:$ For $^{L} \rightarrow\{1,1 / 2,0\}$ tal que

$$
v(\varphi) \in\{1,1 / 2\} \text { sse } b(\varphi)=\mathrm{T}
$$

Prova. Definimos $v$ como segue:
(a) $v(\varphi)=1$ se $b(\varphi)=\mathrm{T}$ e $b(\neg \varphi)=\mathrm{F}$
(b) $v(\varphi)=1 / 2$ se $b(\varphi)=\mathrm{T}$ e $b(\neg \varphi)=\mathrm{T}$
(c) $v(\varphi)=0$ se $b(\varphi)=\mathrm{F}$ e $b(\neg \varphi)=\mathrm{T}$

Provaremos que $v$ é uma valoração de LP. O caso atômico é imediato. Mostraremos agora o caso dos demais conectivos booleanos.

1) $\varphi=\neg \psi$

Se $v(\psi)=1$, então por definição $b(\psi)=\mathrm{T}$ e $b(\neg \psi)=$ F. Pela definição de $b$, obtemos $b(\neg \neg \psi)=\mathrm{T}$. Uma vez que $b(\neg \psi)=\mathrm{F}$ e $b(\neg \neg \psi)=\mathrm{T}$, então $v(\neg \psi)=0$.

Se $v(\psi)=1 / 2$, então por definição $b(\psi)=\mathrm{T}$ e $b(\neg \psi)=\mathrm{T}$. Da definição de $b$, obtemos $b(\neg \neg \psi)=\mathrm{T}, b(\neg \neg \neg \psi)=\mathrm{T}$. Logo, $v(\neg \psi)=1 / 2$.

Se $v(\psi)=0$, então por definição $b(\psi)=\mathrm{F}$ e $b(\neg \psi)=\mathrm{T}$. Pela definição de $b$, obtemos $b(\neg \neg \psi)=$ F e $b(\neg \neg \neg \psi)=$ T. Daqui, $v(\neg \psi)=1$.

2) $\varphi=\psi \rightarrow \gamma$ 
(2.1) Se $v(\psi)=1$ e $v(\gamma)=1$, obtemos por definição $(b(\psi)=\mathrm{T}$ e $b(\neg \psi)$ $=\mathrm{F})$ e $(b(\gamma)=\mathrm{T}$ e $b(\neg \gamma)=\mathrm{F})$. Então $b(\psi \rightarrow \gamma)=\mathrm{T}$ e $b(\neg(\psi \rightarrow \gamma))=$ F. $\log v(\psi \rightarrow \gamma)=1$.

(2.2) Se $v(\psi)=1$ e $v(\gamma)=1 / 2$, obtemos por definição $(b(\psi)=\mathrm{T}$ e $b(\neg \psi)$ $=\mathrm{F})$ e $(b(\gamma)=\mathrm{T}$ e $b(\neg \gamma)=\mathrm{T})$. Então $b(\psi \rightarrow \gamma)=\mathrm{T}$ e $b(\neg(\psi \rightarrow \gamma))=$ T. Logo $v(\psi \rightarrow \gamma)=1 / 2$.

(2.3) Se $v(\psi)=1$ e $v(\gamma)=0$, obtemos por definição $(b(\psi)=$ T e $b(\neg \psi)$ $=\mathrm{F})$ e $(b(\gamma)=\mathrm{F}$ e $b(\neg \gamma)=\mathrm{T})$. Então $b(\psi \rightarrow \gamma)=\mathrm{F}$ e $b(\neg(\psi \rightarrow \gamma))=$ T. $\operatorname{Logo} v(\psi \rightarrow \gamma)=0$.

(2.4) Se $v(\psi)=1 / 2$ e $v(\gamma)=1$, obtemos por definição $(b(\psi)=$ T e $b(\neg \psi)$ $=\mathrm{T})$ e $(b(\gamma)=\mathrm{T}$ e $b(\neg \gamma)=\mathrm{F})$. Então $b(\psi \rightarrow \gamma)=\mathrm{T}$ e $b(\neg(\psi \rightarrow \gamma))=$ F. Logo $v(\psi \rightarrow \gamma)=1$.

(2.5) Se $v(\psi)=1 / 2$ e $v(\gamma)=1 / 2$, obtemos por definição $(b(\psi)=\mathrm{T}$ e $b(\neg \psi)=\mathrm{T})$ e $(b(\gamma)=\mathrm{T}$ e $b(\neg \gamma)=\mathrm{T})$. Então $b(\psi \rightarrow \gamma)=\mathrm{T}$ e $b(\neg(\psi \rightarrow$ $\gamma))=\mathrm{T} . \log 0(\psi \rightarrow \gamma)=1 / 2$.

(2.6) Se $v(\psi)=1 / 2$ e $v(\gamma)=0$, obtemos por definição $(b(\psi)=\mathrm{T}$ e $b(\neg \psi)$ $=\mathrm{T})$ e $(b(\gamma)=\mathrm{F}$ e $b(\neg \gamma)=\mathrm{T})$. Então $b(\psi \rightarrow \gamma)=\mathrm{T}$ e $b(\neg(\psi \rightarrow \gamma))=$ T. $\log v(\psi \rightarrow \gamma)=1 / 2$.

Os casos $(2.7)$ - (2.9) são os casos em que $v(\psi)=0$. Da definição de $b$, é imediato que $b(\neg \psi)=\mathrm{T}$. Claramente $b(\psi \rightarrow \gamma)=\mathrm{T}$ e $b(\neg(\psi \rightarrow \gamma))=\mathrm{F}$. Então $v(\psi \rightarrow \gamma)=1$. Isso conclui a demonstração. Q.E.D.

Teorema 2.5. Dada uma valoração $v \in \operatorname{sem}_{\mathrm{LP}}$, existe uma bivaloração $b: F^{L} \rightarrow\{\mathrm{T}, \mathrm{F}\}$ tal que:

$b(\varphi)=\mathrm{T}$ sse $v(\varphi) \in\{1,1 / 2\}$, para toda $\varphi \in$ For $^{L}$.

Prova. A prova é por indução na complexidade de $\varphi$.

1) $\varphi=p \in A t$ $b(\mathrm{p})=\mathrm{T}$ sse $v(p)=1$ ou $v(p)=1 / 2$ sse $v(p) \in\{1,1 / 2\}$. 
2) $\varphi=\neg \psi$ e $\psi=p$

$b(\neg \mathrm{p})=\mathrm{T}$ sse $b(\mathrm{p})=\mathrm{F}$ ou $b(\mathrm{p})=\mathrm{T}$ sse $v(p)=0$ ou $v(p) \in\{1,1 / 2\}$ sse $v(\neg p) \in\{1,1 / 2\}$.

2.1) $\varphi=\neg \psi$ e $\psi=\neg \gamma$

$b(\neg \neg \gamma)=\mathrm{T}$ sse $b(\gamma)=\mathrm{T}$ sse (H.I.) $v(\gamma) \in\{1,1 / 2\}$ sse $v(\neg \neg \gamma) \in\{1,1 / 2\} .{ }^{11}$

2.2) $\varphi=\neg \psi$ e $\psi=\gamma \rightarrow \beta$

$b(\neg(\gamma \rightarrow \beta))=\mathrm{T}$ sse $b(\gamma)=\mathrm{T}$ e $b(\neg \beta)=\mathrm{T}$ sse $b(\gamma)=\mathrm{T}$ e $(b(\beta)=\mathrm{F}$ ou $b(\beta)=\mathrm{T})$ sse (H.I.) $v(\gamma) \in\{1,1 / 2\}$ e $(v(\beta)=0$ ou $v(\beta) \in\{1,1 / 2\})$ sse $v(\gamma$

$\rightarrow \beta) \in\{0,1 / 2\}$ sse $v(\neg(\gamma \rightarrow \beta)) \in\{1,1 / 2\}$.

3) $\varphi=\gamma \rightarrow \beta$

$b(\gamma \rightarrow \beta)=\mathrm{T}$ sse $b(\neg \gamma)=\mathrm{T}$ ou $b(\beta)=\mathrm{T}$ sse $(b(\gamma)=\mathrm{F}$ ou $b(\gamma)=\mathrm{T})$ ou $b(\beta)=\mathrm{T}$ sse (H.I.) $(v(\gamma)=0$ ou $v(\gamma) \in\{1,1 / 2\})$ ou $v(\beta) \in\{1,1 / 2\}$ sse $v(\gamma$

$\rightarrow \beta) \in\{1,1 / 2\}$. Isso conclui o que pretendemos demonstrar. Q.E.D.

Os Teoremas 2.4 e 2.5 mostram que LP pode ser apresentada a partir de uma semântica bivalente. Como Caleiro et al (CALEIRO, et al, 2007) observam, esse resultado redutivo de Suszko depende da estruturalidade da relação de consequência lógica. Por outro lado, existem reduções mais gerais que não pressupõem estruturalidade. Um exemplo interessante desta última observação são as semânticas bivaloradas de da Costa (DA COSTA, 1974) para a hierarquia de lógicas $C_{n}$, que em geral não possuem semântica multivalorada finita. É importante dizer que as bivalorações de da Costa foram descobertas independentemente das de Suszko. E o fato de não pressupor a estruturalidade da relação de consequência torna os resultados de da Costa mais gerais que os de Suszko. No que diz respeito à construtividade do resultado, Caleiro et al (CALEIRO et al, 2007) observam que o resultado de Suszko não é construtivo no sentido que Suszko não apresenta um método construtivo que permita obter uma semântica bivalorada para cada lógica multivalorada. Além disso, por mais que o resultado segundo o qual toda lógica multivalorada é logicamente bivalorada seja atribuído a Suszko, a prova deste fato encontra-se no livro de Malinowski (MALINOWSKI, 1993). Em (CALEIRO et al, 2007)

\footnotetext{
${ }^{11}$ Hipótese de Indução.
} 
encontramos um método construtivo que nos permite obter, para cada lógica multivalorada $\mathbf{L}$, uma semântica bivalente que caracteriza $\mathbf{L}$.

As semânticas do estilo apresentado na Definição 2.3 são frequentemente criticadas no que diz respeito a sua intuitividade. Por exemplo, ela não explica o processo que nos permitiu obter $b(\varphi)=b(\neg \varphi)=\mathrm{T}$. No caso da semântica matricial a resposta é imediata, pois $v(\varphi)=v(\neg \varphi)$ quando e somente quando $v(\varphi)=1 / 2$. Por outro lado, é possível obter semânticas bivalentes que sejam capazes de explicar o significado das constantes lógicas da linguagem lógica. Por exemplo, Carnielli Lima Marques (CARNIELLI \& LIMA MARQUES, 1999) apresentam as chamadas Semânticas de Sociedades, que são semânticas bivaloradas para lógicas multivaloradas capazes de descrever situações onde dois ou mais agentes diferentes podem aceitar proposições contraditórias, e situações onde dois ou mais agentes diferentes podem rejeitar tanto uma proposição quanto sua contraditória. Em (BEZERRA, 2017) pode ser encontrado tal semântica para LP. A razão pela qual apresento a semântica da Definição 2.3 em detrimento de outra deve-se pelo fato de que meu objetivo é mostrar a simples possibilidade de apresentar uma semântica bivalente para uma lógica multivalorada.

Embora a verificação de tautologias em lógicas caracterizadas por semânticas bivaloradas não seja tão simples quanto a verificação por meio de tabelas de verdade, Caleiro et al (CALEIRO et al, 2010) defendem que essas semânticas possuem aplicações interessantes no estudo de sistemas formais dentre as quais destacamos: (i) tais semânticas dão um procedimento de decisão para lógicas que não possuem semântica finitamente valorada, como é o caso da hierarquia $C_{n}$ anteriormente citadas, bem como outros sistemas lógicos paraconsistentes; (ii) elas facilitam a construção de sistemas de prova, tal como os tablôs semânticos para essas lógicas, o que permite um procedimento uniforme de provas de completude. Essas aplicações mostram que, além de ser uma tese filosófica acerca da multivaloração, a Tese de Suszko mostra-se como uma ferramenta interessante de investigação lógica.

da Costa et al (DA COSTA et al, 1996) levantam críticas à Tese de Suszko tendo como base a multivaloração inferencial de Malinowski (MALI- 
NOWSKI, 1994). Malinowski reconhece que a divisão bipartite do conjunto de valores lógicos implica que a relação de consequência $\vDash$ seja tarskiana estrutural e, portanto, bivalente. Contudo, ele afirma que a multivaloração lógica ainda é possível da seguinte maneira:

The departure is a division of the matrix universe into three subsets of: $r e$ jected elements, accepted elements and all other elements. On such grounds it was possible to define the relation being a formal counterpart of reasoning admitting rules of inference which from non-rejected assumptions lead to accepted conclusions (...) The relation was then called, somewhat inaccurately, a $q$-consequence (...) According to this $\varphi$ is inferred from the set of premisses $X$, whenever it is the case that if all premisses are not rejected then $\varphi$ is accepted. (MALINOWSKI, 2007, p. 68)

Em que sentido podemos dizer que a definição apresentada por Malinowski é um problema para a Tese de Suszko? Essa definição não configura um contraexemplo ao resultado redutivo de Suszko, pois Tsuji (TSUJI, 1998) mostra que a relação de consequência definida por Malinowski não é reflexiva. Portanto, não é tarskiana estrutural e não configura um contraexemplo ao teorema de Suszko. Mesmo assim, da Costa et al argumentam que ela configura um contraexemplo à Tese de Suszko, uma vez que é possível mostrar que a $q$ consequência admite trivalorações lógicas. De fato, Malinowski (MALINOWSKI, 1990) mostra que tais relações de consequência não admitem necessariamente redução a um modelo bivalente. Em virtude dessa possibilidade, da Costa et al argumentam que não temos razão a priori para defender tal tese.

Embora possa reconhecer certo mérito na argumentação de da Costa et al, reconheço que $\vDash_{\mathrm{L}}$ e a q-consequência capturam diferentes aspectos do conceito de consequência lógica. Como podemos ver na citação acima, a q-consequência não é uma relação que preserva valores designados do conjunto de premissas para a conclusão, mas que preserva atitudes proposicionais. Suponha a possibilidade de definir uma variante da q-consequência para o caso de LP de maneira que o valor $1 / 2$ tanto ao conjunto de valores aceitos quanto ao conjunto de valores rejeitados. Chamemos tal relação de $\vDash_{\text {d-LP. }}$ Claramente $\vDash_{\mathrm{LP}} \mathrm{e} \vDash_{\mathrm{d}-\mathrm{LP}}$ capturam diferentes aspectos de LP, podendo ser utilizada 
para capturar aspectos hipotéticos das ciências empíricas. ${ }^{12}$ Consequentemente, $\vDash_{\text {d-LP }}$ perderá ao menos uma das propriedades de $\vDash_{\text {LP. }}$. Sob essa ótica não é claro se LP munida de $\vDash_{\text {d-LP }}$ captura o aspecto de preservação de verdade que $\vDash_{\text {LP }}$ captura. Em suma, $\vDash_{L P}$ e $\vDash_{\text {d-LP }}$ não descrevem as mesmas coisas. A primeira descreve contexto de preservação de verdade enquanto o segundo descreve contextos voltados para hipóteses de ciência empírica. Então, não podemos dizer que uma relação de consequência do tipo $\vDash_{\text {d-LP }}$ é um contraexemplo à Tese de Suszko, que é uma tese alética. A relação de consequência proposta por Malinowski captura aspectos puramente inferenciais de uma lógica. O que a objeção de da Costa et al mostra é que lógicas, de maneira geral, não possuem interpretações canônicas. ${ }^{13}$

O que a Tese de Suszko implica é que, de uma perspectiva alética, a lógica é bivalorada. Aqui não nos comprometeremos com teses metafísicas que a suportam. De um ponto de vista ontológico, pode ser argumentado que existem muitos valores de verdade além do verdadeiro e falso. Por outro lado, uma resposta definitiva a esse problema pode não ser possível. Adotarei uma justificação pragmática a seu favor. $\mathrm{O}$ fato de lógicas não-clássicas terem uma metateoria clássica já é um indício em defesa do valor pragmático da Tese de Suszko. ${ }^{14}$ Além disso, a própria definição de consequência lógica adotada por Suszko em (SUSZKO, 1977) captura intuições importantes presentes na prática argumentativa em matemática. Neste ponto, estou de acordo com Beall \& Restall (BEALL \& RESTALL, 2006) que uma relação de consequência, se entendida como preservadora de verdade do conjunto de premissas para a conclusão, intuitivamente satisfaz as propriedades da relação de consequência

\footnotetext{
${ }^{12}$ Para uma exposição sistemática de relações generalizadas de consequência, convidamos o leitor a consultar a tese de doutoramento de Carolina Blasio (BLASIO, 2017).

${ }^{13}$ A tese segundo a qual uma lógica não possui interpretação canônica não é nova na literatura. Podemos encontrá-la, por exemplo, em (BARRIO, 2018). Nesse artigo, Barrio concentra-se em lógicas paraconsistentes. No presente trabalho me concentro em lógicas multivaloradas, de maneira geral.

${ }^{14} \mathrm{Em}$ (BACON, 2013), Bacon argumenta em favor da possibilidade de definir uma metateoria não-clássica para uma lógica não-clássica. Defendemos, entretanto, que essa possibilidade não constitui necessariamente um contraexemplo para a Tese de Suszko, visto que em muitas lógicas o conceito de verdade pode não possuir papel fundamental, como é o caso de certas lógicas paraconsistentes defendidas por Carnielli \& Rodrigues (CARNIELLI \& RODRIGUES, 2019).
} 
tarskiana. Considere, por exemplo, a seguinte definição informal de consequência lógica:

(*) A é consequência lógica de $\Sigma$ se e somente se em todo caso que as sentenças de $\Sigma$ são verdadeiras, A é verdadeira.

Uma definição no estilo (*) pode ser encontrada em (BEALL \& RESTALL, 2006). A sentença $A$ e o conjunto $\Sigma$ de sentenças estão sendo consideradas como pertencendo à linguagem natural e sendo livres de ambiguidade. A informalidade de (*) deve-se a não especificação da palavra 'caso'. Pode ser facilmente mostrado que a definição (*) satisfaz as propriedades da relação de consequência $\vDash_{\mathbf{L}}$ apresentadas acima. Então, a relação $\vDash_{\mathbf{L}}$ consegue capturar uma noção importante de consequência lógica na linguagem natural. Mas, o fato da relação $\vDash_{\mathbf{L}}$ capturar uma noção de consequência lógica na linguagem natural não significa que ela captura uma noção intuitiva, pré-teórica, de consequência lógica. Como Smith (SMITH, 2011) argumenta, por mais que $\vDash_{\mathbf{L}}$ consiga capturar uma noção informal de consequência lógica, isso não significa dizer que ela captura uma noção pré-teórica/primitiva de consequência lógica. Uma noção tal como (*) é resultado de um refinamento teórico necessário para dizermos que ela se aproxima de uma noção formal de consequência teoricamente relevante como $\vDash_{\mathbf{L}}$. Consequentemente, não podemos dizer que $(*)$ é $a$ noção primitiva de consequência lógica. Isso não constitui um problema visto que nossas noções pré-teóricas podem ser confusas e, até mesmo, errôneas. Como Smith argumenta, é muito difícil precisar qual é a noção pré-teórica de consequência. Possivelmente, ela carrega elementos de relevância e não de preservação de verdade. Mas não sabemos. O que podemos realmente dizer acerca de $(*)$ é que ela consegue descrever uma relação importante de consequência lógica que pode ser usada tanto na linguagem natural quanto no raciocínio matemático. Nesse aspecto, podemos defender a razoabilidade da relação de consequência que sustenta a própria Tese de Suskzo.

Mesmo as lógicas que não são redutíveis a modelos bivalorados não parecem contradizer a Tese de Suzsko um ponto de vista filosófico, pois pode ser argumentado que suas relações de consequência não preservam verdade no 
sentido estrito do termo. Podem, por exemplo, interpretar os valores algébricos como atitudes proposicionais tais como 'aceitação' e 'rejeição' em relação às variáveis proposicionais. Essa pluralidade de relações de consequência correspondentes a uma mesma linguagem não constitui um problema, pois corrobora com a tese segundo a qual uma lógica não possui uma interpretação canônica (BARRIO, 2018). Enquanto sistema formal, uma lógica pode admitir muitas interpretações. Os modelos que apresentamos para LP corrobora com essa afirmação. Mostramos ser possível apresentar uma semântica matricial trivalorada, bem como uma semântica de bivalorações para LP.

\section{2. É a Tese de Suszko um golpe definitivo contra as lógicas multivaloradas?}

Se a Tese de Suszko é razoável, poderíamos nos perguntar se ela é um obstáculo para lógicas multivaloradas. Defendo que essas lógicas podem conviver com a tese segundo a qual existem somente dois valores lógicos. Tal coexistência é possível em virtude do fato de essas lógicas, enquanto sistemas formais, não se reduzirem às semânticas de matrizes. Argumentei anteriormente que a possibilidade de apresentar mais de uma semântica para lógica mostra que não existe uma interpretação canônica para uma lógica. A razão de chamarmos uma lógica $\mathbf{L}$ de $n$-valorada deve-se ao fato de $n$ ser a menor cardinalidade do conjunto de valores de verdade das matrizes que caracterizam L. Por outro lado, não podemos reduzir $\mathbf{L}$ à semântica de matrizes. Nesse sentido tais semânticas podem ser vistas como possíveis representações para essas lógicas. Segundo Marcos (MARCOS, 2009), a não ser que exista um resultado que impeça que uma lógica tenha uma determinada propriedade, é um erro classificá-la tendo em vista a semântica a partir da qual essa lógica é circunstancialmente apresentada. Ou seja, não podemos dizer, do ponto de vista semântico, que uma lógica tem essencialmente uma determinada propriedade já que é a princípio possível dar uma semântica para essa lógica que não tenha essa propriedade, a não ser que a lógica em questão falhe em possuir determinada propriedade. ${ }^{15}$ Por exemplo, não podemos dizer que a semântica

\footnotetext{
15 A lógica intuicionista é um exemplo de lógica que não pode ser apresentada por meio de uma semântica matricial finita. Tal impossibilidade foi demonstrada por Gödel em (GÖ-
} 
matricial de LP nos compromete com um terceiro valor de verdade além do verdadeiro e do falso já que é possível caracterizar essa lógica por meio de uma semântica bivalente na qual esse terceiro valor não existe. Assim, por mais que essas lógicas sejam apresentadas através de uma semântica matricial que admitem mais valores (algébricos) de verdade além do verdadeiro e do falso, elas podem ser representadas por semânticas bivalentes. E elas admitem semânticas bivalentes devido aos resultados redutivos aos quais estão sujeitas. Nesse aspecto, cada semântica se apresenta como uma maneira de explicar o vocabulário de uma lógica L. Do ponto de vista das bivalorações, por exemplo, podemos entender quais são os princípios válidos sobre verdade e falsidade em L. Por essas razões, defendo que as lógicas multivaloradas podem coexistir com a Tese de Suszko.

\section{Pluralismo lógico e Tese de Suszko}

O pluralismo lógico é uma posição filosófica que reconhece a existência de mais de uma lógica como correta. $\mathrm{O}$ debate recente acerca do pluralismo lógico ganhou força a partir de uma série de trabalhos de Beall \& Restall, principalmente com o livro (BEALL \& RESTALL, 2006). Como apresentamos anteriormente, eles propõem a definição informal (*) de consequência lógica que é compatível com mais de uma especificação da palavra 'caso'. Se, por exemplo, substituirmos a 'caso' por 'construções', obtemos a lógica intuicionista. Por outro lado, a substituição da palavra 'caso' por 'modelos,' entendidos como modelos tarskianos para lógica de primeira ordem, obtemos a própria lógica de primeira ordem. Ou seja, diferentes especificações da palavra 'caso' resultam em definições formais de consequência. ${ }^{16}$

Entretanto, uma objeção a qual muitas versões do pluralismo estão sujeitas, inclusive a formulada por Beall \& Restall, é a objeção da mudança de significado. Essa objeção foi formulada por Quine (QUINE, 1986). De acordo

DEL, 1932).

${ }^{16}$ É importante ressaltar que nem toda especificação da palavra 'caso' define uma relação de consequência. Em (BEALL \& RESTALL, 2006), Beall \& Restall defendem que a instância resultante da substituição deve satisfazer alguns critérios (formalidade, necessidade e normatividade) para ser considerada como uma instância de uma relação de consequência. Para mais detalhes, convidamos o leitor a conferir o referido livro. 
com Quine, proponentes de sistemas lógicos substancialmente distintos falam sobre coisas distintas. Por sistemas lógicos substancialmente distintos entendo sistemas que discordam em ao menos um princípio lógico. Sejam $\mathbf{L}_{1}$ e $\mathbf{L}_{2}$ dois sistemas lógicos substancialmente distintos. Consequentemente, ainda que $\mathbf{L}_{1}$ e $\mathbf{L}_{2}$ possuam ao menos um conectivo $c$ em comum, o significado de $c$ em $\mathbf{L}_{1}$ diferirá do significado de $c$ em $\mathbf{L}_{2}{ }^{17}$

A versão do pluralismo com o qual me ocuparei aqui é o pluralismo de relações de consequência proposto por Hjortland (HJORTLAND, 2011). No referido artigo, Hjortland propõe uma versão do pluralismo lógico que ele chama de pluralismo intra-teórico. O pluralismo intra-teórico consiste na presença de mais uma relação de consequência dentro de uma mesma teoria lógica, sendo esta entendida como um par $(L, \Vdash)$, onde $L$ é uma linguagem e $\Vdash$ é uma relação de consequência definida em $L$. Ou seja, Hjortland admite teorias lógicas do tipo $\left(L, \Vdash_{1}, \ldots, \Vdash_{n}\right)$, onde $\Vdash_{1}, \ldots, \mathbb{}_{n}$ denotam relações de consequência distintas definidas na mesma linguagem $L$, ao mesmo tempo em que admite que o significado dos conectivos seja fixo em cada uma das $\Vdash_{1}, \ldots, \mathbb{}_{n}$. Do ponto de vista do cálculo de sequentes, isso significa que as regras dos conectivos são mantidas fixas em todas as relações de consequência, ao passo que as regras estruturais dos sequentes podem variar em cada uma dessas relações. Isso significa que o núcleo de cada conectivo é preservado nas diferentes relações de consequênciạ $\Vdash_{\mathrm{i}}$, para $1 \leq \mathrm{i} \leq \mathrm{n} .{ }^{18}$

Já do ponto de vista semântico, com o qual irei me concentrar aqui, isso significa que o significado dos conectivos é dado pela tabela dos valores algébricos. Então, as tabelas dos valores algébricos são mantidas fixas, ao passo que a variação consiste na escolha dos valores distinguidos em cada uma das relações $\vDash_{\mathrm{i}}$, para $1 \leq \mathrm{i} \leq \mathrm{n}$. Ou seja, a matriz $\mathscr{M}_{\mathrm{i}}$ de cada $\vDash_{\mathrm{i}}$ possuirá o mesmo conjunto de valores algébricos, e as operações possuirão as mesmas tabelas. O que muda, portanto, é o conjunto $D$ de valores distinguidos. Essa versão do pluralismo aplica-se a todas às lógicas multivaloradas. ${ }^{19}$

\footnotetext{
${ }^{17}$ É importante ter em mente que a Quine considera a lógica clássica como lógica ortodoxa, a lógica do discurso científico. A questão da mudança de assunto direciona-se principalmente às lógicas heterodoxas, isto é, às lógicas divergentes da lógica clássica.

18 Para os detalhes sintáticos, convidamos o leitor a conferir (HJORTLAND, 2011).
} 
Considere novamente o exemplo da lógica LP. ${ }^{20}$ Como apresentado anteriormente, sua matriz $\mathscr{M}$ possui o conjunto $\{1,1 / 2\}$ de valores distinguidos. É sabido que a lógica $\mathrm{K}_{3}$ (RESCHER, 1969) é caracterizada pela matriz $\mathscr{M}^{\prime}=(\{1,1 / 2,0\}, \neg, \rightarrow,\{1\})$, onde os operadores $\neg \mathrm{e} \rightarrow$ possuem as mesmas tabelas de valores algébricos que os operadores da matriz $\mathscr{M}$ de LP. Ou seja, assumindo que o significado de um conectivo possa ser dado pela tabela de valores algébricos que o caracteriza, obtém-se que os conectivos de $\mathrm{LP}_{\text {e }} \mathrm{K}_{3}$ possuem o mesmo significado. A diferença entre as lógicas reside nas relações de consequência que as caracterizam. Portanto, das relações de consequência $\vDash_{\mathrm{LP}}$ e $\vDash_{\mathrm{K} 3}$ definidas a partir das matrizes $\mathscr{M}$ e $\mathscr{M}$ ' obtemos uma teoria $\left(L, \vDash_{\mathrm{LP}}\right.$, $\vDash_{\mathrm{K} 3}$ ), onde $L$ é a linguagem comum a LP e $\mathrm{K}_{3}$.

Ocuparei-me agora com a questão de como a Tese de Suszko se relaciona com o pluralismo intra-teórico. Como vimos na subseção 2.2, os Teoremas 2.4 e 2.5 mostram que $\vDash_{L P}=\vDash^{2}{ }_{\text {LP. }}$ É possível tanto definir uma semântica nos moldes da Definição 2.3 quanto provar, utilizando um raciocínio similar, dois teoremas análogos aos Teoremas 2.4 e 2.5 em relação à lógica $\mathrm{K}_{3}$. Ou seja, é possível provar que $\vDash_{\mathrm{K} 3}=\vDash^{2}{ }_{\mathrm{K} 3}$. Haverá, por outro lado, ao menos uma diferença entre as bivalorações de LP e as bivalorações de $\mathrm{K}_{3}$, uma vez que LP é paraconsistente e $\mathrm{K}_{3}$ é paracompleta, isto é, que não valida o princípio do terceiro excluído.

Se o que muda nas matrizes é a escolha dos elementos do conjunto de valores algébricos que tomamos como distinguidos, consequentemente, o que muda nas bivalorações é a escolha da razão da verofuncionalidade da negação. Nas bivalorações de LP, a verdade de $\neg \varphi$ não é determinada pela falsidade de $\varphi$. Por outro lado, nas bivalorações de $\mathrm{K}_{3}$, a falsidade de $\neg \varphi$ não é determina-

\footnotetext{
${ }^{19}$ Uma maneira de introduzir a discussão do pluralismo lógico com respeito às lógicas multivaloradas seria articular a relação entre valores algébricos e lógicas instrumentais, por ex emplo. Na literatura podemos encontrar diversos exemplos interessantes dessa relação. A própria lógica $\mathrm{K}_{3}$ é um exemplo interessante. A proposta de Kleene visava descrever funções recursivas parciais que podem estar indefinidas para determinados objetos em um dado domínio. Por outro lado, a proposta de Kleene não visava apresentar $\mathrm{K}_{3}$ como uma lógica que caracteriza princípios mais gerais do raciocínio. Ou seja, $\mathrm{K}_{3}$ não foi pretendida como uma lógica canônica (PRIEST, 2006b). Os valores intermediários de lógicas multivaloradas, tais como as nonsense-logics (PIRÓG-RZEPECKA, 1977) foram introduzidos como instrumentos para caracterizar certos aspectos de raciocínio que a lógica clássica não trata, ao menos imediatamente. Essa discussão, embora interessante e importante de ser feita, não será abordada em detalhes neste artigo.

${ }^{20}$ Essa mesma lógica é objeto de análise no artigo de Hjortland.
} 
da pela verdade de $\varphi$. De uma perspectiva puramente técnica, as bivalorações explicitam as condições de uma fórmula ter um valor (não-) designado. Seja $D$ o conjunto de valores designados.

(i) $v(\varphi \rightarrow \psi) \in D$ se e somente se $v(\neg \varphi) \in D$ ou $v(\psi) \in D$

(ii) $v(\varphi \rightarrow \psi) \notin D$ sse $v(\varphi) \in D$ e $v(\psi) \notin D$

(iii) $v(\neg(\varphi \rightarrow \psi)) \in D$ sse $v(\varphi) \in D$ e $v(\neg \psi) \in D$

(iv) $v(\neg \neg \varphi) \in D$ sse $v(\varphi) \in D$

(v) se $v(\neg \varphi) \in D$ então $v(\varphi) \notin D$ ou $v(\varphi) \in D$

(vi) se $v(\neg \varphi) \notin D$ então $v(\varphi) \in D$

Já no caso $\mathrm{K}_{3}$, as cláusulas (v) e (vi) são modificadas como segue

(v') se $v(\neg \varphi) \notin D$ então $v(\varphi) \notin D$ ou $v(\varphi) \in D$

(vi') se $v(\neg \varphi) \in D$ então $v(\varphi) \notin D$

As cláusulas acima são descrições mais gerais do que ocorre nas tabelas de verdade de LP e de $\mathrm{K}_{3}$. Os conectivos de ambas as lógicas, portanto, possuem comportamentos similares em cenários distintos.

Note que essa argumentação mostra a compatibilidade do pluralismo intra-teórico com a Tese de Suszko, uma vez que as bivalorações podem ser vistas como explicitando o que apresentamos nos dois últimos parágrafos. Além disso, não parece haver inconsistência entre a Tese de Suszko e a afirmação de que o significado dos conectivos é dado pelas suas tabelas de valores algébricos, uma vez que a semântica de bivalorações e a semântica matricial capturam diferentes aspectos de uma mesma lógica.

Em última instância, a relação entre a pluralidade de lógicas e a Tese de Suszko mostra uma independência do $P N C$, $P T E$ e $P E$ em relação ao princípio de bivalência. A lógica clássica satisfaz todos esses princípios mencionados, o que pode sugerir que eles estão necessariamente interligados. Por outro lado, outras lógicas mais fracas mostram que podemos não ter todos eles conjuntamente e elas possuem legitimidade. Por exemplo, a lógica LP valida 
$P T E, P N C$ mas não valida $P E$. Já a lógica trivalorada $K_{3}$ valida $P E$, mas não valida tanto $P N C$ quanto PTE. Outras lógicas multivaloradas exemplificam que esses três princípios são realmente independentes uns dos outros. A lógica $I^{l}$ valida $P E$ e $P N C$, mas não valida $P T E$. Por último, a lógica $P^{l}$ valida $P T E$, mas não valida $P N C$ e $P E .^{21}$ Diferentes lógicas podem nos mostrar maneiras segundo as quais esses princípios podem relacionar-se.

\section{Considerações finais}

$\mathrm{Na}$ apresentação das bivalorações para LP, foi dito que as semânticas do estilo da Definição 2.3 carecem de intuitividade por não oferecerem uma explicação do significado das constantes lógicas. Por outro lado, podemos entendê-las como estabelecendo normas para a verdade das fórmulas da linguagem de uma determinada lógica. Mencionei a possibilidade apresentar semânticas bivaloradas que são mais intuitivas. ${ }^{22}$ A possibilidade de apresentar semânticas alternativas à semântica matricial permite uma perspectiva interessante às lógicas multivaloradas, que também são frequentemente criticadas por não oferecerem explicações adequadas dos seus valores intermediários. Assim, a variedade de semânticas bivaloradas para essas lógicas nos permite enxergá-las a partir de diferentes perspectivas, abrindo um horizonte de possíveis aplicações.

A Tese de Suszko pode ser vista como uma tese acerca da natureza da lógica, uma vez que ela compreende a lógica como sendo uma teoria da consequência preservadora de verdade. Vimos, contudo, que uma lógica enquanto sistema formal é aberta a diferentes interpretações e que ela não possui interpretação canônica. Por outro lado, defendo que ela é uma tese plausível caso entendamos lógica como sendo interpretada em termos aléticos. E sua compatibilidade com a tese do pluralismo lógico sugere que os próprios valores de

\footnotetext{
${ }^{21}$ Convidamos o leitor a checar o artigo (CARNIELLI \& RODRIGUES, 1999) para uma apresentação das lógicas $P^{l}$ e $I^{l}$.

${ }^{22}$ Uma semântica bivalorada que sequer mencionamos aqui é a semântica de supervalorações, que são capazes de oferecer uma explicação interessante das constantes lógicas. Uma aplicação bem sucedida dessa semântica foi na teoria de Kripke dos pontos fixos como uma solução ao problema dos paradoxos semânticos. Convidamos o leitor a consultar (PRIEST, 2008) para uma apresentação dessa semântica.
} 
verdade são independentes de $P T E, P N C$ e $P E$. Tal independência pode ajudar na compreensão do que são os valores lógicos.

A Tese de Suszko tal como é apresentada é uma tese semântica, uma vez que é enunciada em termos de conceitos puramente semânticos. Consequentemente, a redução à bivaloração concerne à teoria de modelos, foi apresentada uma teoria de modelos para LP que possui somente dois valores que coincide com as matrizes para LP no que diz respeito a suas validades. Uma vez que essas lógicas possuem um sistema dedutivo em relação aos quais é possível demonstrar teoremas de correção e completude, podemos nos perguntar se é possível formular essa tese em termos de conceitos de teoria da prova. Conjecturo que tal formulação é possível se nos atentarmos ao tablôs analíticos para lógicas multivaloradas. Carnielli (CARNIELLI, 1987) apresenta um método para construir sistemas de tablôs para qualquer lógica finitamente valorada $\mathbf{L}_{\mathrm{n}}$. Esses sistemas são rotulados, no sentido de possuírem rótulos que precedem as fórmulas em cada nó do tablô. Além disso, dada uma lógica $\mathbf{L}_{\mathrm{n}}$ seu sistema correspondente de tablôs terá $n$ rótulos. Interessantemente, (CALEIRO et al, 2007) apresenta um método construtivo para obter tablôs que possuem somente dois rótulos para lógicas $\mathbf{L}_{n}$. Caso seja possível apresentar um método redutivo que nos permite obter um sistema de tablôs birrotulados a partir de um sistema de tablôs $n$-rotulados, teremos então uma redução à la Suszko de uma perspectiva sintática. A partir de um procedimento desses será possível formular uma tese filosófica mais geral que a Tese de Suszko, visto que esta última é uma tese particularmente semântica.

\section{Referências}

ASENJO, Florencio G. A calculus of antinomies. Notre Dame Journal of Formal Logic, v. 7, n. 1, p. 103-105, 1966.

BACON, Andrew. Non-classical metatheory for non-classical logics. Journal of Philosophical Logic, v. 42, n. 2, p. 335-355, 2013.

BARRIO, Eduardo Alejandro. Models \& Proofs: LFIs Without a Canonical Interpretations. Principia: an international journal of epistemology, v. 22, n. 1, p. 87-112, 2018. 
BEALL, Jeffrey C.; RESTALL, Greg. Logical pluralism. Oxford University Press on Demand, 2006.

BEZERRA, Edson V. Uma análise das bivalorações do ponto de vista das semânticas de sociedade. Dissertação de mestrado. Universidade Estadual de Campinas, 2017.

BLASIO, Carolina. Sobre noções de consequência generalizadas e lógicas plurivalentes. Tese de doutorado. Universidade Estadual de Campinas. 2017.

CALEIRO, Carlos; CARNIELLI, Walter A.; CONIGLIO, Marcelo; MARCOS, João. Two's company: "The humbug of many logical values". In: Logica universalis. Birkhäuser Basel, 2005. p. 169-189.

CALEIRO, Carlos; MARCOS, Joao. Two Many Values: An Algorithmic Outlook on Suszko's Thesis. In: 2010 40th IEEE International Symposium on Multiple-Valued Logic. IEEE, 2010. p. 93-97.

CARNIELLI, Walter A. Systematization of finite many-valued logics through the method of tableaux. The Journal of Symbolic Logic, v. 52, n. 2, p. 473493, 1987.

CARNIELLI, Walter A. Paul Bernays and the eve of non-standard models in logic. Universal logic: an anthology, 2012.

CARNIELLI, Walter A.; LIMA-MARQUES, Mamede. Society semantics and multiple-valued logics. Advances in Contemporary Logic and Computer Science, v. 235, p. 33-52, 1999.

CARNIELLI, Walter; RODRIGUES, Abilio. An epistemic approach to paraconsistency: a logic of evidence and truth. Synthese, v. 196, n. 9, p. 37893813, 2019.

DA COSTA, Newton CA et al. Malinowski and Suszko on many-valued logics: on the reduction of many-valuedness to two-valuedness. Modern Logic, v. 6, n. 3, p. 272-299, 1996.

DA COSTA, Newton CA. On the theory of inconsistent formal systems. Notre dame journal of formal logic, v. 15, n. 4, p. 497-510, 1974.

GÖDEL, Kurt. Zum intuitionistischen aussagenkalkül. Anzeiger Akademie der Wissenschaften Wien, mathematisch-naturwissenschaftliche Klasse, v. 69 , p. 65-66, 1932.

HJORTLAND, Ole Thomassen. Logical Pluralism, Meaning-Variance and Verbal Disputes. Australasian Journal of Philosophy, v. 91, n. 2, p. 355373, 2013. 
KNEALE, William; KNEALE, Martha. The development of logic. Oxford University Press, 1962.

ŁOŚ, Jerzy; SUSZKO, Roman. Remarks on sentential logics. Indagationes mathematicae, v. 20, n. 2, p. 177-183, 1958.

MALINOWSKI, Grzegorz. Inferential many-valuedness. In: Philosophical logic in Poland. Springer, Dordrecht, 1994. p. 75-84.

MALINOWSKI, Grzegorz. Many-valued logic and its philosophy. The Many-Valued and Nonmonotonic Turn in Logic, v. 8, p. 13-94, 2007.

MALINOWSKI, Grzegorz. Many-Valued Logics. Oxford Logic Guides 25. 1993.

MALINOWSKI, Grzegorz. Non-Fregean Logic and Other Formalizations of Propositional Identity'. Bulletin of the Section of Logic, v. 14, n. 1, p. 2127, 1985.

MALINOWSKI, Grzegorz. Q-consequence operation. Reports on Mathematical Logic, v. 24, p. 49 - 59, 1990.

MARCOS, João. What is a non-truth-functional logic?. Studia Logica, v. 92, n. 2, p. 215, 2009.

PIRÓG-RZEPECKA, Krystyna. Systemy nonsense-logics. Państowe Wydawn. Naukowe, 1977.

PRIEST, Graham. The logic of paradox. Journal of Philosophical logic, v. 8, p. 219-241, 1979.

PRIEST, Graham. In contradiction. Oxford University Press, 2006a.

PRIEST, Graham. Doubt truth to be a liar. Oxford University Press, 2006 b.

PRIEST, Graham. An introduction to non-classical logic: From if to is. Cambridge University Press, 2008.

QUINE, Willard V. Philosophy of logic. Harvard University Press, 1986.

RESCHER, Nicholas. Many-valued logics. McGraw Hill New York, 1969.

SMITH, Peter. Squeezing arguments. Analysis, v. 71, n. 1, p. 22-30, 2011.

SUSZKO, Roman. The Fregean axiom and Polish mathematical logic in the 1920s. Studia Logica: An International Journal for Symbolic Logic, v. 36, n. 4, p. 377-380, 1977. 
Perspectiva Filosófica, vol. 47, n. 2, 2020

TARSKI, Alfred. Logic, semantics, metamathematics: papers from 1923 to 1938. Hackett Publishing, 1983.

TSUJI, Marcelo. Many-valued logics and Suszko's thesis revisited. Studia Logica, v. 60, n. 2, p. 299-309, 1998.

WÓJCICKI, Ryszard. Theory of logical calculi: basic theory of consequence operations. Springer Science \& Business Media, 2013.

WITTGENSTEIN, Ludwig. Tractatus Logico-Philosophicus. Tradução de Luiz Henrique Lopes dos Santos. São Paulo, Edusp, 2010. 